\title{
The $A+B \rightarrow \emptyset$ annihilation reaction in a quenched random velocity field
}

\author{
K Oerding † \\ Department of Physics, Theoretical Physics, 1 Keble Road, Oxford OX1 3NP, UK
}

\begin{abstract}
Using field-theoretic renormalization group methods the long-time behaviour of the $A+B \rightarrow \emptyset$ annihilation reaction with equal initial densities $n_{\mathrm{A}}(0)=n_{\mathrm{B}}(0)=n_{0}$ in a quenched random velocity field is studied. At every point $(x, \mathbf{y})$ of a $d$-dimensional system the velocity is parallel or antiparallel to the $x$-axis and depends on the coordinates perpendicular to the flow. Assuming that $v(\mathbf{y})$ have zero mean and short-range correlations in the y-direction we show that the densities decay asymptotically as $n_{\mathrm{A}, \mathrm{B}}(t) \simeq A n_{0}^{1 / 2} t^{-(d+3) / 8}$ for $d<3$. The amplitude $A$ is calculated at first order in $\epsilon=3-d$.
\end{abstract}

PACS numbers: $05.40 .+\mathrm{j}, 47.70 .-\mathrm{n}, 82.20 .-\mathrm{w}$

Short title: The $A+B \rightarrow \emptyset$ reaction in a random velocity field

December 3, 2017

$\dagger$ Address after 1 December 1996: Institut für Theoretische Physik III, Heinrich-Heine-Universität, D-40225 Düsseldorf, Germany 


\section{Introduction}

It is well known that the time development of reaction diffusion systems in which two types of particles A and B ('particles' and 'antiparticles') annihilate irreversibly

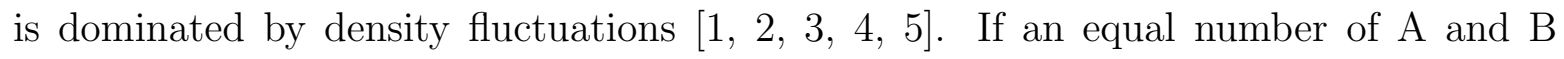
particles is randomly placed on the sites of a $d$-dimensional lattice the densities are asmptotically given by $n(t) \sim t^{-d / 4}$ (for $d<4$ ) whereas a naïve approach based on mean-field rate equations predicts $n(t) \sim t^{-1}$.

The slow density decay is due to the asymptotic segregation of particles into regions of purely $\mathrm{A}$ or $\mathrm{B}$ particles. The reaction becomes more efficient if the effects of segregation are reduced by an appropriate mixing mechanism like turbulence [6] or random forces in low-viscosity liquids [7]. In these cases the diffusive particle motion is replaced by a superdiffusive behaviour characterized by a mean-square displacement of the form $\left\langle x(t)^{2}\right\rangle \sim t^{2 / z}$ with $z<2$. Very recently the annihilation reaction in a driven diffusive system with repulsive interaction between particles of the same type [8, 9] has been studied. If the particles are subject to an external driving force in one direction the repulsion gives rise to superdiffusion with $z=3 / 2$ (for $d=1$ ), and the density decreases asymptotically as $n(t) \sim t^{-1 / 3}$. The relation between the long-time behaviour of $n(t)$ and the value of the exponent $z$ has been investigated for particles performing independent Lévy walks [10]. In this case $z$ is defined through the scaling of the transition probability (which is a function of $r^{-z} t$ ) since the the mean-square displacement for Lévy flights with $z<2$ diverges. It has been shown that below the upper critical dimension $d_{c}(z)=2 z$ the long-time behaviour of the density is given by $n(t) \sim t^{-d /(2 z)}$.

The mixing caused by the inhomogeneous velocity field in a linear shear flow reduces the critical dimension to $d_{c}=2$, i.e. $n(t) \sim t^{-1}$ in any physically accessible dimension [11]. Other models for particle mixing have been considered in references [12, 13]. In this paper we investigate the $\mathrm{A}+\mathrm{B}$-annihilation reaction in a medium with a random velocity field. It is assumed that the velocity at every point $\mathbf{r}=(x, \mathbf{y})$ of a $d$-dimensional system is parallel or antiparallel to the $x$-axis and depends only on the coordinates perpendicular to the flow. The velocity field is modelled by quenched Gaussian random variables with zero mean and the correlations

$$
\left[v(\mathbf{y}) v\left(\mathbf{y}^{\prime}\right)\right]=f \delta\left(\mathbf{y}-\mathbf{y}^{\prime}\right)
$$

where the square brackets indicate the average over the realizations of the flow. Originally this model was introduced to describe the ground water transport in heterogeneous rocks 14.

Below three dimensions a random walk in the presence of a velocity field of the form (1) displays superdiffusive behaviour in the $x$-direction [15, 16]. For a particle starting at $\mathbf{r}=\mathbf{0}$ at time $t=0$ the mean square displacement in the $x$-direction averaged over the configurations of $v(\mathbf{y})$ is given by $\left[\left\langle x^{2}\right\rangle\right] \simeq \sigma^{2} t^{(5-d) / 2}$ for $d<3$ (with a generalized 
diffusion constant $\sigma^{2}$ ). One can use this result for a naïve estimate of the densities at large times $t$. If we approximate the motion of the particles by independent anisotropic Lévy flights with exponents $z_{\|}=4 /(5-d)$ (in $x$-direction) and $z_{\perp}=2$ a straightforward generalization of the arguments given in [10] yields

$$
n_{\mathrm{A}, \mathrm{B}}(t) \sim\left[n_{0} t^{-1 / z_{\|}} t^{-(d-1) / z_{\perp}}\right]^{1 / 2}=n_{0}^{1 / 2} t^{-(d+3) / 8}
$$

where $n_{0}$ is the initial density of each particle type. However, it is not clear in how far the effects of the velocity field can be described by independent Lévy walks since $v(\mathbf{y})$ is a quenched random variable with an infinite correlation length in the $x$-direction. In the following sections we will use the field theoretic methods developed in [17, [4] to show that the power law (2) is correct for $t \rightarrow \infty$ and calculate the amplitude at first order in $\epsilon=3-d$. The result of this calculation reads

$$
n_{\mathrm{A}, \mathrm{B}}(t) \simeq A(\epsilon)\left(2 \pi^{3 / 2}(8 \pi D)^{(d-1) / 2} \sigma\right)^{-1 / 2} \sqrt{n_{0}} t^{-(d+3) / 8}
$$

where

$$
A(\epsilon)=1+\frac{\epsilon}{8}(3 \ln 2-1)+\mathrm{O}\left(\epsilon^{2}\right)
$$

and $n_{0}=n_{\mathrm{A}}(0)=n_{\mathrm{B}}(0)$ is the initial density of each particle type. In contrast to the case of annihilating Lévy particles which can be treated in a mean field approximation with random initial conditions the correlated particle motion in a quenched velocity field requires the application of the renormalization group.

In the next section a microscopic model for a reaction diffusion system in a random velocity field is defined and its mapping to a continuum field theory is discussed. In Section 3 we briefly review the renormalized field theory for non-interacting particles in a random velocity field. The results are used in Section 4 to derive an effective action which describes the long-time behaviour of the system. The asymptotic decay of the densities is calculated in section 5 and the results are discussed in Section 6 .

\section{The model}

At every time $t$ the microscopic state of the system is defined by the occupation numbers $m\left(\mathbf{r}_{i}\right)$ of A-particles and $n\left(\mathbf{r}_{i}\right)$ of B-particles at every site $i$ (with position $\mathbf{r}_{i}$ ) of a $d$ dimensional lattice. Denoting the probability for a given configuration $\{m, n\}$ at time $t$ by $P(\{m, n\} ; t)$ we can describe the stochastic dynamics of the system by a set of linear differential equations (master equation) for the probabilities $P(\{m, n\} ; t)$. (We assume that the time evolution is Markoffian.) In order to apply field theoretic renormalization group methods we use a functional integral description which is equivalent to the master equation. Since a detailed derivation of this formalism is given in references [17, 18, [4] we only give the main steps focussing on the modifications that are necessary to study the influence of the random velocity field. 
We first map the probabilities $P(\{m, n\} ; t)$ to a vector $|\Phi(t)\rangle$ of the infinite dimensional Fock space spanned by the vectors $|\{m, n\}\rangle$ by writing [19, 20]

$$
|\Phi(t)\rangle=\sum_{\{m, n\}} P(\{m, n\} ; t)|\{m, n\}\rangle .
$$

Since the master equation is linear and of first order in $t$ it may be written in the form

$$
\partial_{t}|\Phi(t)\rangle=\hat{L}|\Phi(t)\rangle
$$

with an appropriate Liouville operator $\hat{L}$. This operator can be expressed in terms of the annihilation operators $\hat{a}_{\mathrm{A}}\left(\mathbf{r}_{i}\right), \hat{a}_{\mathrm{B}}\left(\mathbf{r}_{i}\right)$ and creation operators $\hat{a}_{\mathrm{A}}^{\star}\left(\mathbf{r}_{i}\right), \hat{a}_{\mathrm{B}}^{\star}\left(\mathbf{r}_{i}\right)$ defined by

$$
\begin{array}{ll}
\hat{a}_{\mathrm{A}}\left(\mathbf{r}_{i}\right)\left|m\left(\mathbf{r}_{i}\right)\right\rangle=m\left(\mathbf{r}_{i}\right)\left|m\left(\mathbf{r}_{i}\right)-1\right\rangle & \hat{a}_{\mathrm{A}}^{\star}\left(\mathbf{r}_{i}\right)\left|m\left(\mathbf{r}_{i}\right)\right\rangle=\left|m\left(\mathbf{r}_{i}\right)+1\right\rangle \\
\hat{a}_{\mathrm{B}}\left(\mathbf{r}_{i}\right)\left|n\left(\mathbf{r}_{i}\right)\right\rangle=n\left(\mathbf{r}_{i}\right)\left|n\left(\mathbf{r}_{i}\right)-1\right\rangle & \hat{a}_{\mathrm{B}}^{\star}\left(\mathbf{r}_{i}\right)\left|n\left(\mathbf{r}_{i}\right)\right\rangle=\left|n\left(\mathbf{r}_{i}\right)+1\right\rangle .
\end{array}
$$

These operators leave the occupation numbers $m\left(\mathbf{r}_{j}\right)$ and $n\left(\mathbf{r}_{j}\right)$ of the sites $j \neq i$ unchanged.

The diffusion of non-interacting particles corresponds to the Liouvillean

$$
\begin{aligned}
\hat{L}_{D}=\frac{D}{h^{2}} \sum_{<i, j>} & \left(\hat{a}_{\mathrm{A}}^{\star}\left(\mathbf{r}_{j}\right)-\hat{a}_{\mathrm{A}}^{\star}\left(\mathbf{r}_{i}\right)\right)\left(\hat{a}_{\mathrm{A}}\left(\mathbf{r}_{i}\right)-\hat{a}_{\mathrm{A}}\left(\mathbf{r}_{j}\right)\right) \\
& +\frac{D}{h^{2}} \sum_{<i, j>}\left(\hat{a}_{\mathrm{B}}^{\star}\left(\mathbf{r}_{j}\right)-\hat{a}_{\mathrm{B}}^{\star}\left(\mathbf{r}_{i}\right)\right)\left(\hat{a}_{\mathrm{B}}\left(\mathbf{r}_{i}\right)-\hat{a}_{\mathrm{B}}\left(\mathbf{r}_{j}\right)\right)
\end{aligned}
$$

where the sum extends over all pairs of nearest neighbour sites, $h$ is the lattice spacing and $D$ denotes the diffusion constant. The annihilation of particle-antiparticle pairs at the same site is described by

$$
\hat{L}_{\text {reac }}=\frac{k}{h^{d}} \sum_{i}\left(1-\hat{a}_{\mathrm{A}}^{\star}\left(\mathbf{r}_{i}\right) \hat{a}_{\mathrm{B}}^{\star}\left(\mathbf{r}_{i}\right)\right) \hat{a}_{\mathrm{A}}\left(\mathbf{r}_{i}\right) \hat{a}_{\mathrm{B}}\left(\mathbf{r}_{i}\right)
$$

where $k / h^{d}$ is the reaction rate.

To investigate the effect of a random shear flow we introduce independent Gaussian random variables $v(\mathbf{y})$ labelled by the coordinates $\mathbf{y}$ perpendicular to the direction of the flow. At each point $\mathbf{r}_{i}=\left(x_{i}, \mathbf{y}_{i}\right)$ the velocity field prefers particle jumps parallel or antiparallel to the $x$-direction depending on the sign of $v\left(\mathbf{y}_{i}\right)$. If $v\left(\mathbf{y}_{i}\right)$ is positive particles at the point $\left(x_{i}, \mathbf{y}_{i}\right)$ will jump with rate $v\left(\mathbf{y}_{i}\right) / h$ in positive $x$-direction whereas for $v\left(\mathbf{y}_{i}\right)<0$ they will hop with rate $-v\left(\mathbf{y}_{i}\right) / h$ in the opposite direction. Here we assume that $v(\mathbf{y})$ have zero mean and the correlations

$$
\left[v(\mathbf{y}) v\left(\mathbf{y}^{\prime}\right)\right]=\bar{f} h^{-(d-1)} \delta_{\mathbf{y}, \mathbf{y}^{\prime}} .
$$

While deviations from the Gaussian distribution turn out to be irrelevant for the asymptotic scaling behaviour long range correlations of the velocity field [21] or a nonvanishing mean shear [1] change the universality class. The motion of the particles in 
the velocity field is described be the Liouvillean

$$
\begin{aligned}
\hat{L}_{\text {flow }}=\frac{1}{h} \sum_{i} \mid & v\left(\mathbf{y}_{i}\right) \mid\left[\hat{a}_{\mathrm{A}}^{\star}\left(\mathbf{r}_{i}+\operatorname{sgn}\left(v\left(\mathbf{y}_{i}\right)\right) h \mathbf{e}_{x}\right)-\hat{a}_{\mathrm{A}}^{\star}\left(\mathbf{r}_{i}\right)\right] \hat{a}_{\mathrm{A}}\left(\mathbf{r}_{i}\right) \\
+ & \frac{1}{h} \sum_{i}\left|v\left(\mathbf{y}_{i}\right)\right|\left[\hat{a}_{\mathrm{B}}^{\star}\left(\mathbf{r}_{i}+\operatorname{sgn}\left(v\left(\mathbf{y}_{i}\right)\right) h \mathbf{e}_{x}\right)-\hat{a}_{\mathrm{B}}^{\star}\left(\mathbf{r}_{i}\right)\right] \hat{a}_{\mathrm{B}}\left(\mathbf{r}_{i}\right)
\end{aligned}
$$

where $\operatorname{sgn}(v)= \pm 1$ is the sign function and $\mathbf{e}_{x}$ denotes the unit vector in positive $x$-direction. In order to perform the average over the realizations of $v(\mathbf{y})$ it will be convenient to write $\hat{L}_{\text {flow }}$ as a sum, $\hat{L}_{\text {flow }}=\hat{L}^{(-)}+\hat{L}^{(+)}$, where

$$
\begin{array}{rl}
\hat{L}^{(-)}=\frac{1}{2 h} \sum_{i} & v\left(\mathbf{y}_{i}\right)\left[\hat{a}_{\mathrm{A}}^{\star}\left(\mathbf{r}_{i}+h \mathbf{e}_{x}\right)-\hat{a}_{\mathrm{A}}^{\star}\left(\mathbf{r}_{i}-h \mathbf{e}_{x}\right)\right] \hat{a}_{\mathrm{A}}\left(\mathbf{r}_{i}\right) \\
& +\frac{1}{2 h} \sum_{i} v\left(\mathbf{y}_{i}\right)\left[\hat{a}_{\mathrm{B}}^{\star}\left(\mathbf{r}_{i}+h \mathbf{e}_{x}\right)-\hat{a}_{\mathrm{B}}^{\star}\left(\mathbf{r}_{i}-h \mathbf{e}_{x}\right)\right] \hat{a}_{\mathrm{B}}\left(\mathbf{r}_{i}\right)
\end{array}
$$

is odd with respect to the velocity field and

$$
\begin{aligned}
\hat{L}^{(+)}=\frac{1}{2 h} \sum_{i} & \left|v\left(\mathbf{y}_{i}\right)\right|\left[\hat{a}_{\mathrm{A}}^{\star}\left(\mathbf{r}_{i}+h \mathbf{e}_{x}\right)+\hat{a}_{\mathrm{A}}^{\star}\left(\mathbf{r}_{i}-h \mathbf{e}_{x}\right)-2 \hat{a}_{\mathrm{A}}^{\star}\left(\mathbf{r}_{i}\right)\right] \hat{a}_{\mathrm{A}}\left(\mathbf{r}_{i}\right) \\
& +\frac{1}{2 h} \sum_{i}\left|v\left(\mathbf{y}_{i}\right)\right|\left[\hat{a}_{\mathrm{B}}^{\star}\left(\mathbf{r}_{i}+h \mathbf{e}_{x}\right)+\hat{a}_{\mathrm{B}}^{\star}\left(\mathbf{r}_{i}-h \mathbf{e}_{x}\right)-2 \hat{a}_{\mathrm{B}}^{\star}\left(\mathbf{r}_{i}\right)\right] \hat{a}_{\mathrm{B}}\left(\mathbf{r}_{i}\right)
\end{aligned}
$$

depends only on the modulus $|v(\mathbf{y})|$. The even part $\hat{L}^{(+)}$can be interpreted as a contribution to the diffusion in $x$-direction with a $\mathbf{y}$-dependent diffusion constant.

The formal solution of equation (6) is given by

$$
|\Phi(t)\rangle=\exp (\hat{L} t)|\Phi(0)\rangle
$$

with $\hat{L}=\hat{L}_{\text {diff }}+\hat{L}_{\text {reac }}+\hat{L}_{\text {flow }}$. In order to derive a functional integral representation for the dynamics one uses the Trotter formula

$$
\exp (\hat{L} t)=\lim _{n \rightarrow \infty}(1+\hat{L} t / n)^{n}
$$

to rewrite the time evolution operator as a product of $\mathrm{n}$ factors linear in $\hat{L}$. Inserting the identity operator in a coherent state representation between the individual factors [17, [8, 4] one obtains a path integral with action

$$
\begin{aligned}
S[\tilde{a}, a ; \tilde{b}, b]= & h^{d} \int \mathrm{d} t \sum_{i}\left[\tilde{a}\left(\mathbf{r}_{i}, t\right) \partial_{t} a\left(\mathbf{r}_{i}, t\right)+\tilde{b}\left(\mathbf{r}_{i}, t\right) \partial_{t} b\left(\mathbf{r}_{i}, t\right)\right] \\
& -\int \mathrm{d} t L[\tilde{a}(t), a(t) ; \tilde{b}(t), b(t)]-h^{d} \sum_{i} n_{0}\left(\tilde{a}_{\mathrm{A}}\left(\mathbf{r}_{i}, 0\right)+\tilde{a}_{\mathrm{B}}\left(\mathbf{r}_{i}, 0\right)\right)
\end{aligned}
$$

where $\tilde{a}, a, \tilde{b}$ and $b$ are c-number functions and $L[\tilde{a}(t), a(t) ; \tilde{b}(t), b(t)]$ can be obtained from $\hat{L}$ via the replacements

$$
\begin{array}{ll}
\hat{a}_{\mathrm{A}}\left(\mathbf{r}_{i}\right) \rightarrow h^{d} a\left(\mathbf{r}_{i}, t\right) & \hat{a}_{\mathrm{A}}^{\star}\left(\mathbf{r}_{i}\right) \rightarrow 1+\tilde{a}\left(\mathbf{r}_{i}, t\right) \\
\hat{a}_{\mathrm{B}}\left(\mathbf{r}_{i}\right) \rightarrow h^{d} b\left(\mathbf{r}_{i}, t\right) & \hat{a}_{\mathrm{B}}^{\star}\left(\mathbf{r}_{i}\right) \rightarrow 1+\tilde{b}\left(\mathbf{r}_{i}, t\right) .
\end{array}
$$


In equation (16) $n_{0}$ denotes the initial density of each species.

In order to calculate the average of correlation and response functions with respect to disorder we use the effective action $\bar{S}$ defined by

$$
\exp (-\bar{S}[\tilde{a}, a ; \tilde{b}, b])=[\exp (-S[\tilde{a}, a ; \tilde{b}, b])]
$$

For each value of the coordinate $\mathbf{y}$ one has to calculate an integral of the form

$$
\int_{-\infty}^{\infty} d v(\mathbf{y})\left(2 \pi \bar{f} / h^{d-1}\right)^{-1 / 2} \exp \left(\frac{v(\mathbf{y})^{2}}{2 \bar{f} / h^{d-1}}\right) \cdot \exp (-A v(\mathbf{y})-B|v(\mathbf{y})|)
$$

where $A$ and $B$ are shorthand notations for the contributions to $S$ coming from $\hat{L}^{(-)}$ and $\hat{L}^{(+)}$, respectively. The integral (19) can be expressed in terms of error functions. We only need the approximation

$$
\text { (19) }=\exp \left(\frac{1}{2}\left(\bar{f} / h^{d-1}\right) A^{2}-\sqrt{\frac{2}{\pi}}\left(\bar{f} / h^{d-1}\right)^{1 / 2} B+\ldots\right)
$$

since higher orders in $A$ and $B$ turn out to be irrelavant for the asymptotic scaling behaviour. The first term in the exponential function (proportional to $A^{2}$ ) gives rise to a new interaction which is non-local with respect to time while the second contribution (linear in $B$ ) modifies the diffusion constant in the direction parallel to the flow. Neglecting all irrelevant terms one arrives at

$$
\begin{aligned}
\bar{S}[\tilde{a}, a ; \tilde{b}, b]= & \int \mathrm{d} t \int \mathrm{d}^{d} r\left[\tilde{a}\left(\partial_{t} a-D \triangle_{\perp} a-D_{\|} \partial_{\|}^{2} a\right)\right. \\
& \left.+\tilde{b}\left(\partial_{t} b-D \triangle_{\perp} b-D_{\|} \partial_{\|}^{2} b\right)+k a b(\tilde{a}+\tilde{b}+\tilde{a} \tilde{b})-n_{0}(\tilde{a}+\tilde{b}) \delta(t)\right] \\
& -\frac{\bar{f}}{2} \int \mathrm{d}^{d-1} y\left(\int \mathrm{d} t \int \mathrm{d} x\left(\tilde{a} \partial_{\|} a+\tilde{b} \partial_{\|} b\right)\right)^{2} .
\end{aligned}
$$

Here the sum over lattice sites has been replaced by an integral over the continuous variable $\mathbf{r}=\left(r_{\|}, \mathbf{r}_{\perp}\right)=(x, \mathbf{y})$. This continuum model is appropriate for the study of the long-time and large-distance behaviour of the system.

Since the quantity $\psi=(a-b) / \sqrt{2}$ is closely related to the conserved density difference of A-particles and B-particles it is convenient to introduce the fields [4

$\psi=\frac{1}{\sqrt{2}}(a-b) \quad \tilde{\psi}=\frac{1}{\sqrt{2}}(\tilde{a}-\tilde{b}) \quad \phi=\frac{1}{\sqrt{2}}(a+b) \quad \tilde{\phi}=\frac{1}{\sqrt{2}}(\tilde{a}+\tilde{b})$.

After rescaling of time by the diffusion constant $D$ the action becomes in terms of the new variables

$$
\begin{aligned}
\bar{S}[\tilde{\psi}, \psi ; \tilde{\phi}, \phi]= & \int \mathrm{d} t \int \mathrm{d}^{d} r\left[\tilde{\psi}\left(\partial_{t} \psi-\triangle_{\perp} \psi-\kappa \partial_{\|}^{2} \psi\right)+\tilde{\phi}\left(\partial_{t} \phi-\triangle_{\perp} \phi-\kappa \partial_{\|}^{2} \phi\right)\right. \\
& \left.+\lambda_{1} \tilde{\phi}\left(\phi^{2}-\psi^{2}\right)+\lambda_{2}\left(\tilde{\phi}^{2}-\tilde{\psi}^{2}\right)\left(\phi^{2}-\psi^{2}\right)-\sqrt{2} n_{0} \tilde{\phi} \delta(t)\right] \\
& -\frac{f}{2} \int \mathrm{d}^{d-1} y\left(\int \mathrm{d} t \int \mathrm{d} x\left(\tilde{\psi} \partial_{\|} \psi+\tilde{\phi} \partial_{\|} \phi\right)\right)^{2}
\end{aligned}
$$


with the coupling coefficients $\lambda_{1}=k /(\sqrt{2} D), \lambda_{2}=k /(4 D), f=\bar{f} / D^{2}$ and $\kappa=D_{\|} / D$.

The action $\bar{S}$ includes two different types of fluctuations. The interaction proportional to $\lambda_{2}$ leads to anticorrelations neglected in the mean field rate equations. In the case of the single species annihilation $A+A \rightarrow \emptyset$ these anticorrelations are responsible for the slow density decay $n(t) \sim t^{-d / 2}$ in $d<2$ [18]. The interaction proportional to $f$ describes the effect of the quenched random velocity field and gives rise to the superdiffusive motion of particles found in references [16, 15]. A straightforward dimensional analysis shows that $\lambda_{2}$ is relevant below $d_{\lambda}=2$ while the upper critical dimension of the disorder is $d_{f}=3$. As already pointed out in 四 this does not mean that $\lambda_{2}$ may be neglected from the outset if $d>d_{\lambda}$. For non-vanishing $\lambda_{2}$ coarse graining of the action $\bar{S}$ generates new interactions which are relevant below four dimensions. Since these interactions are located at the 'time surface' $t=0$ they modify the initial distribution of the fields $\psi$ and $\phi$. An effective action appropriate for the analysis of fluctuation effects in dimensions $d>d_{\lambda}$ includes all relevant interactions which may be generated by the renormalization group for $\lambda_{2}>0$.

We will use renormalization group improved perturbation theory to derive results from the effective action for non-zero $f$. Since this calculation is based on the results of reference [15] for non-interacting particles (i.e. $\lambda_{1}=\lambda_{2}=0$ ) it is useful to review the renormalization group approach to this problem briefly in the next section.

\section{Non-interacting particles in a random velocity field}

For $\lambda_{1}=\lambda_{2}=n_{0}=0$ only Green functions of the form

$$
G_{M, N}(\{\mathbf{r}, t\})=\left.\left\langle\prod_{i=1}^{M} \psi\left(\mathbf{r}_{i}, t_{i}\right) \tilde{\psi}\left(\tilde{\mathbf{r}}_{i}, \tilde{t}_{i}\right) \cdot \prod_{j=M+1}^{M+N} \phi\left(\mathbf{r}_{j}, t_{j}\right) \tilde{\phi}\left(\tilde{\mathbf{r}}_{j}, \tilde{t}_{j}\right)\right\rangle\right|_{\lambda=0}
$$

with an equal number of insertions of $\psi(\phi)$ and $\tilde{\psi}(\tilde{\phi})$ are non-zero. (Throughout this section the angular brackets indicate an average with weight $\exp (-\bar{S})$ for $\lambda_{1}=\lambda_{2}=$ $n_{0}=0$.)

Since $f$ is a relevant parameter below three dimensions the asymptotic scaling behaviour of the Green functions cannot be obtained from a naïve perturbation expansion at finite order in $f$. We therefore use the renormalization group to improve the perturbation theory by a partial summation of the perturbation series. Due to ultraviolet-divergencies at the upper critical dimension $d_{f}=3$ the individual contributions to the perturbation series have poles in $\epsilon=3-d$. In the minimal renormalization scheme these poles are absorbed into renormalizations of coupling coefficients and fields. 
The propagator of the free (Gaussian) field theory is given by

$$
\langle\psi(\mathbf{r}, t) \tilde{\psi}(\mathbf{0}, 0)\rangle_{0}=\langle\phi(\mathbf{r}, t) \tilde{\phi}(\mathbf{0}, 0)\rangle_{0}=\int_{\mathbf{q}} \mathrm{e}^{\mathrm{iq \mathbf {q }}} G(\mathbf{q}, t)
$$

with the Fourier transform

$$
G(\mathbf{q}, t)=\Theta(t) \exp \left(-\left(q_{\perp}^{2}+\kappa q_{\|}^{2}\right) t\right)
$$

Here $\Theta(t)$ is the Heaviside step function and the indices ' $\|$ ' and ' $\perp$ ' indicate the respective directions parallel and perpendicular to the flow. Due to the strong anisotropy of the velocity field the theory is superrenormalizable in the upper critical dimension [15], i.e. the number of superficially divergent diagrams is finite. In fact, it is sufficient to subtract the $\epsilon$-pole of the diagram shown in figure 1 to render the field theory finite at every order of the perturbation theory. The required renormalization factor can be obtained from the response function $G_{1,0}=G_{0,1}$ at one-loop order. A straightforward calculation yields

$$
\int \mathrm{d}^{d} r \exp \left(-\mathrm{i} q_{\|} r_{\|}\right) G_{1,0}(\mathbf{r}, t)=\exp \left(-\kappa q_{\|}^{2} t\right)\left[1-\frac{2 f}{\epsilon(1+\epsilon / 2)(4 \pi)^{1-\epsilon / 2}} q_{\|}^{2} t^{1+\epsilon / 2}+\mathrm{O}\left(f^{2} q_{\|}^{4}\right)\right] .
$$

To subtract the $\epsilon$-pole in this function we introduce the renormalized diffusion constant $\kappa_{R}=Z^{-1} \kappa$, where

$$
Z=1-\frac{2 u}{\epsilon} \quad \text { and } \quad A_{\epsilon} f=\kappa_{R} u \mu^{\epsilon} .
$$

Here $u$ is a renormalized coupling coefficient, $A_{\epsilon}=1 /\left((4 \pi)^{1-\epsilon / 2}(1+\epsilon / 2)\right)$ is a geometrical factor and $\mu$ denotes an external momentum scale.

Since the bare Green function is independent of $\mu$ its derivative at fixed bare parameters vanishes, i.e.

$$
\left.\mu \frac{\mathrm{d}}{\mathrm{d} \mu}\right|_{0} G_{M, N}(\{\mathbf{r}, t\} ; \kappa, f)=0 .
$$

Figure 1. The only superficially divergent Feynman diagram of the field theory defined by the action $\bar{S}$ [equation (23)] with $\lambda_{1}=\lambda_{2}=n_{0}=0$. The full line with an arrow represents the propagator $(26)$ and the wavy line corresponds to the correlator $f \delta\left(\mathbf{r}_{\perp}-\mathbf{r}_{\perp}^{\prime}\right)$ of the velocity field.

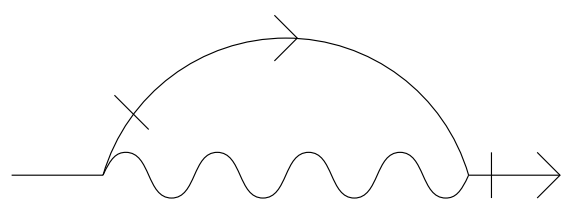


Expressing $\kappa$ and $f$ in terms of $\kappa_{R}, u$ and $\mu$ we obtain for the renormalized Green function the renormalization group equation (RGE)

$$
\left[\mu \partial_{\mu}-\zeta(u) \kappa_{\mathrm{R}} \partial_{\kappa_{\mathrm{R}}}+\beta(u) \partial_{u}\right] G_{M, N}\left(\{\mathbf{r}, t\} ; \kappa_{R}, u ; \mu\right)=0 .
$$

Due to superrenormalizability the Wilson functions

$$
\zeta(u)=\left.\mu \frac{\mathrm{d}}{\mathrm{d} \mu}\right|_{0} \ln Z=2 u \quad \beta(u)=\left.\mu \frac{\mathrm{d}}{\mathrm{d} \mu}\right|_{0} u=u(-\epsilon+2 u)
$$

are exact at every order of the perturbation theory. Solving the RGE by characteristics one finds

$$
G_{M, N}\left(\{\mathbf{r}, t\} ; \kappa_{R}, u ; \mu\right)=G_{M, N}\left(\{\mathbf{r}, t\} ; Y(l) \kappa_{R}, \bar{u}(l) ; \mu l\right)
$$

where

$l \frac{\mathrm{d}}{\mathrm{d} l} \ln Y(l)=-\zeta(\bar{u}(l)) \quad l \frac{\mathrm{d}}{\mathrm{d} l} \bar{u}(l)=\beta(\bar{u}(l)) \quad Y(1)=1 \quad \bar{u}(1)=u$.

For $l \rightarrow 0$ the scale dependent coupling constant $\bar{u}(l)$ tends to the fixed point $u_{\star}=\epsilon / 2$ and $Y(l) \simeq Y_{\star} l^{-\epsilon}$ (with a non-universal scaling factor $Y_{\star}$ ). The asymptotic scaling behaviour of the Green functions follows from equation (32) and dimensional analysis. The result reads (for $l \rightarrow 0$ )

$$
\begin{aligned}
G_{M, N}\left(\{\mathbf{r}, t\} ; \kappa_{R}, u ; \mu\right) & \simeq\left(\mu^{d}\left(Y_{\star} \kappa_{R}\right)^{-1 / 2} l^{d+\epsilon / 2}\right)^{M+N} \\
\times G_{M, N} & \left(\left\{\mu\left(Y_{\star} \kappa_{R}\right)^{-1 / 2} l^{1+\epsilon / 2} r_{\|}, \mu l \mathbf{r}_{\perp}, \mu^{2} l^{2} t\right\} ; 1, u_{\star} ; 1\right) .
\end{aligned}
$$

This equation shows that anomalous scaling dimensions of the variables and fields are given by

$r_{\perp} \sim l^{-1} \quad r_{\|} \sim l^{-(1+\epsilon / 2)} \quad t \sim l^{-2} \quad \tilde{\psi} \psi \sim l^{d+\epsilon / 2} \quad \tilde{\phi} \phi \sim l^{d+\epsilon / 2}$.

The asymptotic form of the Green functions (34) depends on the arbitrary momentum scale $\mu$ and the non-universal amplitude $Y_{\star}$. A combination of these parameters occuring in (34) can be expressed by a quantity that is accessible to experiments or simulations [16]. Consider the random walk of a particle starting at time $t=0$ at the point $\mathbf{r}=\mathbf{0}$. The mean square displacement of the particle in $r_{\|}$-direction averaged over the realizations of the velocity field reads

$$
X(t)^{2}=\int \mathrm{d}^{d} r G_{1,0}(\mathbf{r}, t) r_{\|}^{2} .
$$

Using the one-loop result (27) and equation (34) with $l=\left(\mu^{2} t\right)^{-1 / 2}$ it is easy to show that (for $t \rightarrow \infty$ )

$$
X(t) \simeq \sigma t^{1 / 2+\epsilon / 4} \quad \text { with } \quad \sigma=\sqrt{2}\left(\kappa_{R} Y_{\star}\right)^{1 / 2} \mu^{\epsilon / 2}\left[1+\mathrm{O}\left(\epsilon^{2}\right)\right] .
$$

In the following sections we will always use the parameter $\sigma$ instead of $Y_{\star}$. Due to the strong anisotropy of the velocity field it is also possible to calculate $X(t)$ directly from 
equation (27) without application of the renormalization group. Since the $n^{\text {th }}$ order contribution to $G_{1,0}$ is proportional to $q_{\|}^{2 n}$ and $X(t)^{2}$ is proportional to the derivative of $G_{1,0}$ with respect to $q_{\|}^{2}$ at $\mathbf{q}=\mathbf{0}$ the one-loop result is sufficient to obtain the function $X(t)$ exactly:

$$
X(t)^{2}=2 \kappa t+\frac{8 f}{\epsilon(2+\epsilon)(4 \pi)^{1-\epsilon / 2}} t^{1+\epsilon / 2} .
$$

For $\epsilon=1$ this result was first given by Bouchaud et al [16].

For $d=3(\epsilon=0)$ an ultraviolet cut-off $\Lambda$ is required to regularize the singularity in equation (38). In this case the mean sqare displacement is asymptotically given by

$$
X(t)^{2} \simeq \frac{f}{2 \pi} t \ln \left(\Lambda^{2} t\right) .
$$

\section{Effective action below three dimensions}

The effect of the $\lambda_{2}$-vertex in equation (23) on the long-time behaviour of a reaction diffusion system without velocity field can be described by interactions located at the 'time surface' $t=0$. In reference [4] it was shown by dimensional analysis that an interaction of the type

$$
\left.\int \mathrm{d}^{d} r \frac{1}{m ! n !} \Delta_{m, n} \tilde{\psi}^{m} \tilde{\phi}^{n}\right|_{t=0}
$$

is relevant below $d_{m, n}=2(m+n) /(m+n-1)$ dimensions. $\left(\Delta_{1,0}\right.$ and $\Delta_{0,1}$ are relevant in any dimension.) The critical dimension of $\Delta_{m, n}$ is changed if the motion of the particles is not purely diffusive, e.g. if the particles are subject to a linear shear flow [11. In order to see how the values of $d_{m, n}$ are changed by the random velocity field consider the expansion of $\langle\phi(t)\rangle$ in powers of $\lambda_{1}, \lambda_{2}$ and $f$. For $d<d_{f}=3\left(d>d_{f}\right)$ the contributions to this expansion at a given order in $\lambda_{1}$ and $\lambda_{2}$ become more important (less important) for large $t$ if we increase the order in $f$. Therefore the long-time behaviour of $\langle\phi(t)\rangle$ for $d>3$ is dominated by the diffusive motion of the particles and we may set $f=0$. Below three dimensions we have to retain all orders in $f$ to determine the asymptotic decay of the density. In this case the many-particle response functions (24) play the same role as the Gaussian propagotor (26) in the case $f=0$. It is clear that it is not possible to sum the the power series in $f$ exactly but the renormalization group allows us a partial summation and gives the correct scaling behaviour.

We first determine the scaling dimension of $\lambda_{2}$ for a non-vanishing velocity field below three dimensions. Using the anomalous scaling dimensions given in (35) one finds $\lambda_{2} \sim l^{2-d-\epsilon / 2}=l^{(1-d) / 2}$, i.e. $\lambda_{2}$ is irrelevant for $d>1$. In the same way we obtain

$$
\lambda_{1} \psi \sim l^{2} \quad \lambda_{1} \phi \sim l^{2} \quad \lambda_{1}^{-1} \tilde{\psi} \sim l^{d-2+\epsilon / 2} \quad \lambda_{1}^{-1} \tilde{\phi} \sim l^{d-2+\epsilon / 2}
$$

and the dimension of the initial term

$$
\lambda_{1}^{m+n} \Delta_{m, n} \sim l^{(1-m-n)(d+\epsilon / 2)+2(m+n)} .
$$


In $d=3$ the vertices with $m+n<3$ are relevant. For $m+n \geq 3$ the initial coupling $\Delta_{m, n}$ is relevant below $d_{m, n}=(m+n+3) /(m+n-1)$ dimensions. However, we will show below that the only initial couplings which are generated by the coarse graining procedure employed in reference [4] are $\Delta_{2,0}$ and $\Delta_{0,2}$ (in addition to $\Delta_{0,1}=\sqrt{2} n_{0}$ ), and these couplings are given by $\Delta_{2,0}=-\Delta_{0,2}=n_{0}$ at every order in $\lambda_{2}$.

Since the coupling coefficient $\lambda_{2}$ is irrelevant for $d>1$ one has to introduce a cutoff wave number $\Lambda$ to avoid ultraviolet-divergencies in the perturbation series. The cut-off is required to regularize the diagrams shown in figure 2 which contribute to an effective coupling constant $\lambda_{2 \text {,eff }}$. The Green functions $G_{M, N}$ considered in the previous section occur in these diagrams as subintegrals (represented by rectangles). As in the previous section we calculate these subintegrals without cut-off since they are ultravioletconvergent for $d<3$. After summation of the subdiagrams (using the renormalization group) the cut-off is introduced to perform the remaining integrations. If the cut-off wave number is not too large [an estimate based on equation (38) with $t \sim \Lambda^{-2}$ gives $\left.\Lambda^{\epsilon} \ll f /(\kappa \epsilon)\right]$ the scaling behaviour (34) still holds on the momentum scale of $\Lambda$. In this case the effective coupling has the scaling form $\lambda_{2 \text {,eff }}=\lambda_{2} F_{\lambda}\left(\lambda_{2} \Lambda^{(d-1) / 2} / \sigma\right)$. In the same way coarse graining leads to an effective coupling coefficient $\lambda_{1, \text { eff }}=\lambda_{1} F_{\lambda}\left(\lambda_{2} \Lambda^{(d-1) / 2} / \sigma\right)$ (with the same scaling function $F_{\lambda}$ ). Evaluating the diagrams depicted in figure (2) by renormalization group improved pertubation theory it is possible to compute the coefficients of the Taylor expansion of $F_{\lambda}$ in an $\epsilon$-expansion. [Due to the scaling behaviour (34) of the response functions the diagrams are (infrared-) finite for $d>1$ in the limit of zero external momentum and frequency.] However, this is not very interesting since the result strongly depends on the type of the cut-off.

The initial coupling $\Delta_{2,0}$ is more interesting since it is known to determine (at least for systems without random velocity field) the amplitude of $\langle\phi(t)\rangle$ [ [⿴囗十 . For the

Figure 2. Contributions to the effective coupling coefficient $\lambda_{2, \text { eff }}$ to third order in $\lambda_{2}$. The hatched rectangles represent the Green functions $G_{M, N}$ [equation (24)], where $M$ $(N)$ is the number of broken lines (full lines) running into the rectangle. The direction of each line is indicated by an arrow and reflects causality, i.e. each line points into the direction of larger time arguments.
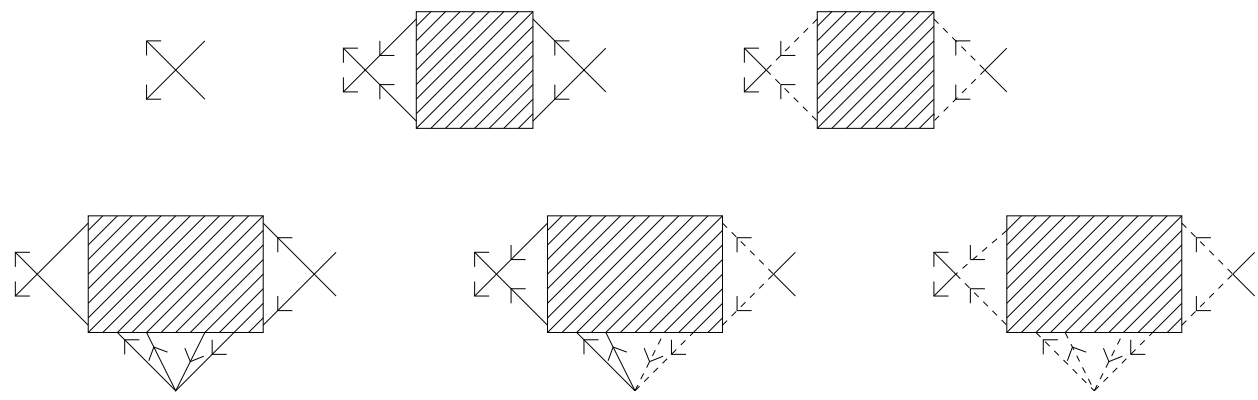
calculation of $\Delta_{2,0}$ it is convenient to shift the field $\phi$ by the mean field density

$$
\Phi_{\mathrm{mf}}(t)=\Phi_{0}\left(1+\lambda_{1} \Phi_{0} t\right)^{-1}
$$

with the initial value $\Phi_{0}=\sqrt{2} n_{0}$. The shifted action $\bar{S}[\tilde{\psi}, \psi ; \tilde{\phi}, \Phi(t)+\phi]$ consists of a Gaussian part

$$
\begin{array}{r}
\bar{S}_{G}[\tilde{\psi}, \psi ; \tilde{\phi}, \phi ; \Phi(t)]=\int \mathrm{d} t \int \mathrm{d}^{d} r\left[\tilde{\psi}\left(\partial_{t} \psi-\triangle_{\perp} \psi-\kappa \partial_{\|}^{2} \psi\right)\right. \\
\left.+\tilde{\phi}\left(\partial_{t} \phi-\triangle_{\perp} \phi-\kappa \partial_{\|}^{2} \phi+2 \lambda_{1} \Phi_{\mathrm{mf}}(t) \phi\right)\right]
\end{array}
$$

and higher order terms summarized in

$$
\begin{gathered}
\bar{S}_{\mathrm{int}}[\tilde{\psi}, \psi ; \tilde{\phi}, \phi ; \Phi(t)]=\int \mathrm{d} t \int \mathrm{d}^{d} r\left[\lambda_{1} \tilde{\phi}\left(\phi^{2}-\psi^{2}\right)+\lambda_{2}\left(2 \Phi_{\mathrm{mf}}(t) \phi+\Phi_{\mathrm{mf}}(t)^{2}\right)\left(\tilde{\phi}^{2}-\tilde{\psi}^{2}\right)\right. \\
\left.+\lambda_{2}\left(\tilde{\phi}^{2}-\tilde{\psi}^{2}\right)\left(\phi^{2}-\psi^{2}\right)\right]-\frac{f}{2} \int \mathrm{d}^{d-1} y\left(\int \mathrm{d} t \int \mathrm{d} x\left(\tilde{\psi} \partial_{\|} \psi+\tilde{\phi} \partial_{\|} \phi\right)\right)^{2}
\end{gathered}
$$

Since $\bar{S}_{G}$ is independent of $f$ the Gaussian (tree) propagators are the same as in

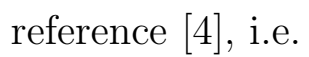

$G_{\psi}\left(\mathbf{q} ; t, t^{\prime}\right)=\int \mathrm{d}^{d} r \mathrm{e}^{-\mathrm{iqr}}\left\langle\psi(\mathbf{r}, t) \tilde{\psi}\left(\mathbf{0}, t^{\prime}\right)\right\rangle_{\mathrm{G}}=G\left(\mathbf{q} ; t-t^{\prime}\right)$
$G_{\phi}\left(\mathbf{q} ; t, t^{\prime}\right)=\int \mathrm{d}^{d} r \mathrm{e}^{-\mathrm{iqr}}\left\langle\phi(\mathbf{r}, t) \tilde{\phi}\left(\mathbf{0}, t^{\prime}\right)\right\rangle_{\mathrm{G}}=\left(\frac{1+\lambda_{1} \Phi_{0} t^{\prime}}{1+\lambda_{1} \Phi_{0} t}\right)^{2} G\left(\mathbf{q} ; t-t^{\prime}\right)$

where $G(\mathbf{q} ; t)$ is the propagator defined in equation (26). Corrections to the mean field density can now be calculated in a diagramatic expansion around the Gaussian action $\bar{S}_{G}$ treating the interactions in $\bar{S}_{\text {int }}$ as perturbations.

Following the lines taken in reference $\left[\right.$ 国 we replace the irrelevant parameter $\lambda_{2}$ by effective initial couplings of the form (40). The diagrams contributing to $\Delta_{m, n}$ have $(m+n)$ external legs each of which is associated with one of the vertices proportional to $\lambda_{2}$. Figure 3 shows the general form of the contributions to $\Delta_{2,0}$. Since the function $\Phi_{\mathrm{mf}}(t)^{2}$ is damped on time scales which are large compared to $\left(\lambda_{1} \Phi_{0}\right)^{-1}$ its effect on the long-time behaviour of the field theory can be described by a $\delta$-function. We may therefore write

$$
\lambda_{2} \Phi_{\mathrm{mf}}(t)^{2} \tilde{\psi}^{2} \simeq \lambda_{2}\left[\int_{0}^{\infty} \mathrm{d} t^{\prime} \Phi_{\mathrm{mf}}\left(t^{\prime}\right)^{2}\right] \delta(t) \tilde{\psi}^{2}=\frac{\lambda_{2}}{\lambda_{1}} \Phi_{0} \delta(t) \tilde{\psi}^{2}
$$

and obtain $\Delta_{2,0}=2 \lambda_{2} \Phi_{0} / \lambda_{1}+\mathrm{O}\left(\lambda_{2}^{2}\right)$. In the same way higher order contributions to $\Delta_{2,0}$ can be calculated by integrating the diagrams (b-e) in figure 3 with respect to the time argument associated with the leftmost vertex. However, the diagrams (b) and (c) cancel, and the same is true for the diagrams (d) and (e). In order to see this we write the contribution of the graph $3(b)$ in the form

$$
\operatorname{graph} \operatorname{3}(\mathrm{b})=\int_{0}^{\infty} \mathrm{d} t\left(-\lambda_{2}\right) f(t)
$$


where $t$ is the time argument carried by the leftmost vertex, and the function $f(t)$ represents the subdiagrams in the hatched bubble and the loop integration. The same function $f(t)$ occurs in the contribution 3 (c) which is given by

$$
\begin{aligned}
\operatorname{graph} 3(\mathrm{c}) & =\int_{0}^{\infty} \mathrm{d} t 2 \lambda_{2} \Phi_{\mathrm{mf}}(t) \int_{0}^{t} \mathrm{~d} t^{\prime} G_{\phi}\left(\mathbf{q}=\mathbf{0} ; t, t^{\prime}\right) \lambda_{1} f\left(t^{\prime}\right) \\
& =2 \lambda_{1} \lambda_{2} \Phi_{0} \int_{0}^{\infty} \mathrm{d} t^{\prime} \int_{t^{\prime}}^{\infty} \mathrm{d} t \frac{\left(1+\lambda_{1} \Phi_{0} t^{\prime}\right)^{2}}{\left(1+\lambda_{1} \Phi_{0} t\right)^{3}} f\left(t^{\prime}\right) \\
& =\int_{0}^{\infty} \mathrm{d} t^{\prime} \lambda_{2} f\left(t^{\prime}\right) .
\end{aligned}
$$

Therefore the contributions (b) and (c) cancel. Analogously one can show that the sum of the diagrams (d) and (e) vanishes. Since the same line of arguments applies if the hatched bubbles in figure 3 have additional external legst, all effective interactions $\Delta_{m, n}$ with $m+n>2$ are zero, and the only non-vanishing initial couplings are given by $\Delta_{2,0}=-\Delta_{0,2}=2 \lambda_{2} \Phi_{0} / \lambda_{1}=n_{0}$. In the appendix of reference [4] a non-zero correction to $\Delta_{2,0}$ of the order $\mathrm{O}\left(\lambda_{2}^{2} n_{0}^{d / 2}\right)$ was obtained because the diagrams of the form (c) and (e) were incorrectly thought to be accounted for by taking $\lambda_{1}$ to $\lambda_{\text {eff }}$.

$\dagger$ Due to causality each diagram which contributes to the initial couplings contains at least one pair of $\tilde{\psi}$ - or $\tilde{\phi}$ - legs coming from a single $\lambda_{2}$ vertex. This is the vertex explicitely shown in figure 3 .

Figure 3. General form of the contributions to $\Delta_{2,0}$. The full and broken bold lines represent the propagators $G_{\phi}$ and $G_{\psi}$, respectively, and the zigzag line corresponds to the mean field density $\Phi_{\mathrm{mf}}(t)$. At lowest order in $\lambda_{2}$ the bubbles in the graphs (b) and (c) contain only the graph (a) as a subdiagram. The vertex proportional to $\Phi_{\mathrm{mf}}(t)$ in the diagrams (c) and (e) is generated by the shift of the field $\phi$ [see equation (45)].

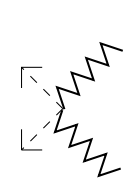

(a)

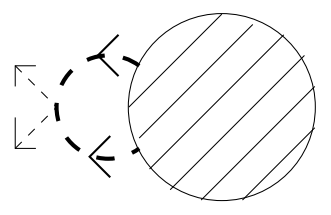

(b)

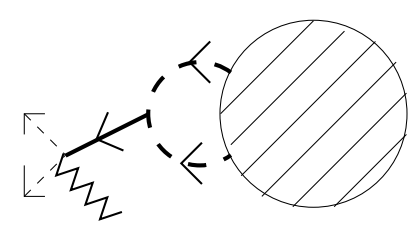

(c)

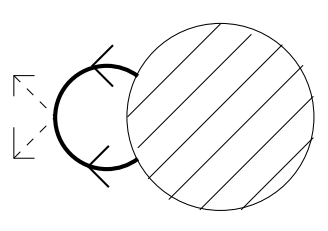

(d)

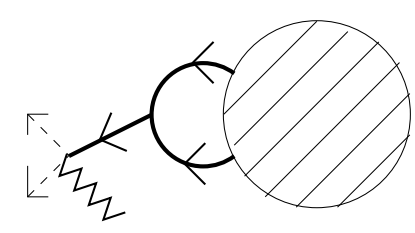

(e) 
The cancellation of diagrams suggests that there should be a simpler way to calculate the initial coupling coefficients. In fact, the effective action can be derived without using Feynman diagrams. This formal derivation exploits the equation of motion

$$
\int D[\tilde{\psi}, \psi ; \tilde{\phi}, \phi] \frac{\delta}{\delta \tilde{\psi}(\mathbf{r}, t)} \exp (-S[\tilde{\psi}, \psi ; \tilde{\phi}, \phi])=0
$$

and the corresponding equation with a functional derivative with respect to $\tilde{\phi}$. The explicit form of the equations of motion (which are valid after insertion into averages) is given by

$\partial_{t} \psi-\triangle_{\perp} \psi-\kappa \partial_{\|}^{2} \psi-2 \lambda_{2} \tilde{\psi}\left(\phi^{2}-\psi^{2}\right)-f\left(\partial_{\|} \psi\right) \int \mathrm{d} t^{\prime} \int \mathrm{d} x^{\prime}\left(\tilde{\psi} \partial_{\|} \psi+\tilde{\phi} \partial_{\|} \phi\right)=0$

and

$$
\begin{gathered}
\partial_{t} \phi-\triangle_{\perp} \phi-\kappa \partial_{\|}^{2} \phi+\lambda_{1}\left(\phi^{2}-\psi^{2}\right)+2 \lambda_{2} \tilde{\phi}\left(\phi^{2}-\psi^{2}\right)-\Phi_{0} \delta(t) \\
-f\left(\partial_{\|} \phi\right) \int \mathrm{d} t^{\prime} \int \mathrm{d} x^{\prime}\left(\tilde{\psi} \partial_{\|} \psi+\tilde{\phi} \partial_{\|} \phi\right)=0 .
\end{gathered}
$$

Averaging (53) one finds

$$
\partial_{t} \Phi(t)+\lambda_{1}\left\langle\phi(\mathbf{r}, t)^{2}-\psi(\mathbf{r}, t)^{2}\right\rangle=\Phi_{0} \delta(t)
$$

where $\Phi(t)=\langle\phi(\mathbf{r}, t)\rangle$ is the exact density. (The equal time averages $\left\langle\tilde{\phi} \psi^{2}\right\rangle$ and $\left\langle\tilde{\phi} \phi^{2}\right\rangle$ are zero due to the prepoint time discretization used in the derivation of the action.) Integration of equation (54) over t yields

$$
\lambda_{1} \int_{0}^{\infty} \mathrm{d} t\left\langle\phi(\mathbf{r}, t)^{2}-\psi(\mathbf{r}, t)^{2}\right\rangle=\Phi_{0} .
$$

The initial fluctuations generated by the irrelevant $\lambda_{2}$ coupling can now be obtained by the replacement

$$
2 \lambda_{2}\left(\phi^{2}-\psi^{2}\right) \longrightarrow 2 \lambda_{2} \delta(t) \int_{0}^{\infty} \mathrm{d} t^{\prime}\left\langle\phi\left(\mathbf{r}, t^{\prime}\right)^{2}-\psi\left(\mathbf{r}, t^{\prime}\right)^{2}\right\rangle=n_{0} \delta(t)
$$

in the equations of motion (52) and (53). The effective equations of motion derived in this way are equivalent to the effective action with the initial vertices $\Delta_{m, n}$ calculated above.

\section{Asymptotic decay of the density}

In order to calculate the long-time behaviour of $\langle\phi(t)\rangle$ we start from the effective action $\bar{S}_{\text {eff }}=\bar{S}_{\text {reac }}+S_{\text {ini }}$, where

$$
\begin{aligned}
\bar{S}_{\text {reac }}[\tilde{\psi}, \psi ; \tilde{\phi}, \phi] & =\int \mathrm{d} t \int \mathrm{d}^{d} r\left[\tilde{\psi}\left(\partial_{t} \psi-\triangle_{\perp} \psi-\kappa \partial_{\|} \psi\right)+\tilde{\phi}\left(\partial_{t} \phi-\triangle_{\perp} \phi-\kappa \partial_{\|} \phi\right)\right. \\
& \left.+\lambda_{1} \tilde{\phi}\left(\phi^{2}-\psi^{2}\right)\right]-\frac{f}{2} \int \mathrm{d}^{d-1} y\left(\int \mathrm{d} t \int \mathrm{d} x\left(\tilde{\psi} \partial_{\|} \psi+\tilde{\phi} \partial_{\|} \phi\right)\right)^{2}
\end{aligned}
$$


and

$$
S_{\mathrm{ini}}[\tilde{\psi}, \tilde{\phi}]=-\int \mathrm{d}^{d} r\left[\Phi_{0} \tilde{\phi}+\frac{1}{2} n_{0}\left(\tilde{\psi}^{2}-\tilde{\phi}^{2}\right)\right]_{t=0} .
$$

At this point it is convenient to reintroduce the Gaussian velocity field $v(\mathbf{y})$ (with $[v(\mathbf{y})]=0$ and $\left[v(\mathbf{y}) v\left(\mathbf{y}^{\prime}\right)\right]=f \delta\left(\mathbf{y}-\mathbf{y}^{\prime}\right)$ as in section 2) to replace $\bar{S}_{\text {reac }}$ in favour of a $v$-dependent action which is local with repect to time. This new action is equivalent to the equations

$$
\begin{aligned}
& \partial_{t} \psi-\triangle_{\perp} \psi-\kappa \partial_{\|}^{2} \psi+v(\mathbf{y}) \partial_{\|} \psi=0 \\
& \partial_{t} \phi-\triangle_{\perp} \phi-\kappa \partial_{\|}^{2} \phi+v(\mathbf{y}) \partial_{\|} \phi+\lambda\left(\phi^{2}-\psi^{2}\right)=0
\end{aligned}
$$

where $\psi$ and $\phi$ are now real fields. The solutions $\psi, \phi$ have to be averaged with respect to both the initial conditions and the realizations of the velocity field. Since the initial state defined by $S_{\mathrm{ini}}$ and the distribution of $v(\mathbf{y})$ are homogeneous the averages of the spatial derivatives in $(59,60)$ vanish. Averaging equation (60) we find

$\dot{\Phi}(t)+\lambda\left(\Phi(t)^{2}-\left\langle\psi(\mathbf{r}, t)^{2}\right\rangle+C(t)\right)=0 \quad$ where $\quad C(t)=\left\langle(\phi(\mathbf{r}, t)-\Phi(t))^{2}\right\rangle$.

To obtain the asymptotic solution of this equation one first has calculate $\left\langle\psi(\mathbf{r}, t)^{2}\right\rangle$ for large $t$. Later in this section we will also need higher moments $\left\langle\psi^{2 n}\right\rangle$ with $n=1,2 \ldots$. Since in equation (59) the dynamics of $\psi$ is decoupled from the reaction process the moments of $\psi$ may be calculated with the Green functions (24). In this way we get

$$
\begin{aligned}
\left\langle\psi(\mathbf{r}, t)^{2 n}\right\rangle & =\left.\frac{1}{2^{n} n !} n_{0}^{n} \int \mathrm{d}^{d} r_{1}^{\prime} \ldots \int \mathrm{d}^{d} r_{n}^{\prime}\left\langle\psi(\mathbf{r}, t)^{2 n} \tilde{\psi}\left(\mathbf{r}_{1}^{\prime}, 0\right)^{2} \ldots \tilde{\psi}\left(\mathbf{r}_{n}^{\prime}, 0\right)^{2}\right\rangle\right|_{\lambda=0} \\
& \simeq \text { const } \times\left(\frac{n_{0}}{\sigma} t^{-(d+3) / 4}\right)^{n}
\end{aligned}
$$

where the scaling form (34) has been used. [A simple power counting as in reference [15] shows that insertions of composite fields such as $\psi^{2 n}$ in equation (62) require no additional renormalizations.]

To obtain the amplitude of the second moment $\left\langle\psi^{2}\right\rangle$ at first order in $\epsilon$ one has to calculate the diagrams shown in figure 6 . The result reads in terms of the unrenormalized coupling constants

$$
\left\langle\psi(\mathbf{r}, t)^{2}\right\rangle=n_{0} \kappa^{-1 / 2}(8 \pi t)^{-d / 2}\left[1-\frac{f t^{\epsilon / 2}}{(4 \pi)^{1-\epsilon / 2} \kappa}\left(\frac{1}{\epsilon}-\frac{3}{2} \ln 2+\mathrm{O}(\epsilon)\right)+\mathrm{O}\left(f^{2}\right)\right] .
$$

Upon application of the renormalization group this becomes (for large $t$ )

$$
\left\langle\psi(\mathbf{r}, t)^{2}\right\rangle \simeq n_{0} \frac{\sqrt{2}}{(8 \pi)^{d / 2} \sigma} t^{-(d+3) / 4}\left[1+\frac{\epsilon}{4}(3 \ln 2-1)+\mathrm{O}\left(\epsilon^{2}\right)\right] .
$$

We are now in a position to determine the asymptotic decay of $\Phi(t)$ in a similar way as in the case $v(\mathbf{y}) \equiv 0$ [4]: 
We first solve equation (61) for $C(t)=0$, i.e. we are looking for a function $\Phi_{\mathrm{u}}(t)$ satisfying

$$
\dot{\Phi}_{\mathrm{u}}(t)+\lambda\left(\Phi_{\mathrm{u}}(t)^{2}-\left\langle\psi(\mathbf{r}, t)^{2}\right\rangle\right)=0 .
$$

Since $\left\langle\psi^{2}\right\rangle=$ cst $\times t^{-(d+3) / 4}$ for $t \rightarrow \infty$ (with $\left.(d+3) / 4<2\right)$ the asymptotic solution of this equation is given by $\Phi_{\mathrm{u}}(t) \simeq \sqrt{\operatorname{cst}} \times t^{-(d+3) / 8}$. Using the positivity of $C(t)$ one can show [0] that $\Phi_{\mathrm{u}}(t)$ is an upper bound for $\Phi(t)$. To derive a lower bound $\Phi_{1}(t) \leq \Phi(t)$ we use the fact that $a=(\phi+\psi) / \sqrt{2}$ and $b=(\phi-\psi) / \sqrt{2}$ are real densities which satisfy the equations of motion

$$
\begin{aligned}
& \partial_{t} a-\triangle_{\perp} a-\kappa \partial_{\|}^{2} a+v(\mathbf{y}) \partial_{\|} a+\sqrt{2} \lambda a b=0 \\
& \partial_{t} b-\triangle_{\perp} b-\kappa \partial_{\|}^{2} b+v(\mathbf{y}) \partial_{\|} b+\sqrt{2} \lambda a b=0 .
\end{aligned}
$$

For non-negative initial values the densities remain non-negative for all $t$. This implies $\phi^{2}-\psi^{2}=2 a b \geq 0$ and leads to the lower bound $\Phi_{1}(t)=\langle|\psi|\rangle$.

Although the initial distribution of $\psi$ is Gaussian, the velocity field generates for $t>0$ higher cumulants in the distribution $\mathcal{P}_{t}$ of $\psi(\mathbf{r}, t)$. We perform a cumulant expansion for this distribution to calculate $\langle|\psi|\rangle$. Since higher cumulants are generated by the disorder vertex $f$ (or its renormalized counterpart $v$ with fixed point value $\left.v_{\star}=\epsilon / 2\right)$ this amounts to an $\epsilon$-expansion for $\mathcal{P}_{t}(\psi)$. The Fourier transform $\tilde{\mathcal{P}}_{t}$ of the distribution with respect to $\psi$ can be written as

$$
\tilde{\mathcal{P}}_{t}(h)=\int_{-\infty}^{\infty} \mathrm{d} \psi \mathcal{P}_{t}(\psi) \mathrm{e}^{\mathrm{i} h \psi}=\exp \left(\sum_{n=1}^{\infty} \frac{c_{2 n}(t)}{(2 n) !}(-1)^{n} h^{2 n}\right)
$$

where $c_{2 n}(t)$ denotes the connected part (cumulant) of the $(2 n)^{\text {th }}$ moment of $\psi$, e.g.

$$
c_{2}(t)=\left\langle\psi(\mathbf{r}, t)^{2}\right\rangle \quad c_{4}(t)=\left\langle\psi(\mathbf{r}, t)^{4}\right\rangle-3 c_{2}(t)^{2} \quad \text { etc. }
$$

For a Gaussian distribution all $c_{m}(t)$ with $m>2$ vanish. Equation(67) can be used to calculate $\langle|\psi|\rangle$ in an expansion in $c_{2 n}(t), n=4,6, \ldots$ :

$\langle|\psi(\mathbf{r}, t)|\rangle=\int_{-\infty}^{\infty} \mathrm{d} \psi|\psi| \int_{-\infty}^{\infty} \frac{\mathrm{d} h}{2 \pi} \tilde{\mathcal{P}}_{t}(h) \mathrm{e}^{-\mathrm{i} h \psi}$

Figure 4. Contributions to $\left\langle\psi^{2}\right\rangle$ at first order in $\epsilon$. The full and empty circles represent the initial vertex $\Delta_{2,0}$ and the field $\psi^{2}$, respectively. A solid line with an arrow corresponds to the Gaussian propagator (26).
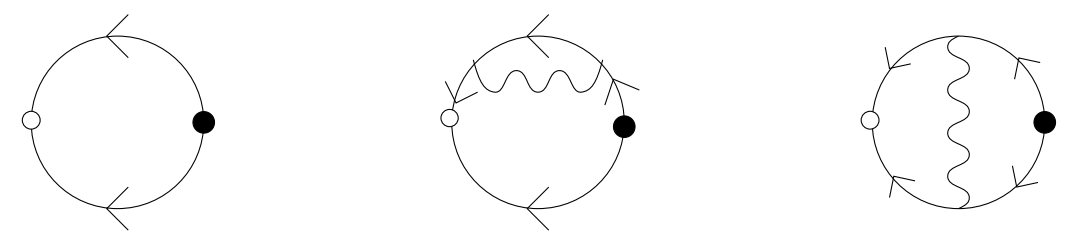


$$
=\sqrt{\frac{2 c_{2}(t)}{\pi}}\left[1-\frac{1}{4 !} \frac{c_{4}(t)}{c_{2}(t)^{2}}+\mathrm{O}\left(c_{4}(t)^{2}, c_{6}(t), \ldots\right)\right] .
$$

Due to the simple scaling $c_{2 n}(t) \sim t^{-n(d+3) / 4}$ of the cumulants for large $t$ the expression in the square brackets tends to a constant. The first correction to the Gaussian distribution comes from the four-point cumulant $c_{4}(t)$. The contributions to $c_{4}(t)$ at second order in $f$ are shown in figure 5 (b-d). The first order (figure 5 (a)) vanishes due to the $x$-derivative associated with the vertex $f$. This means that upon application of the renormalization group the amplitude of $c_{4}(t)$ at the fixed point is of the order $\epsilon^{2}$. Analogously any cumulant $c_{2 n}(t)$ with $n \geq 2$ is of the order $\epsilon^{n}$ since a non-vanishing contribution to this function requires at least $n$ vertices proportional to $f$. Therefore we only need the Gaussian part of the distribution to calculate $\langle|\psi|\rangle$ at first order in $\epsilon$. The result is

$$
\Phi_{1}(t)=\left(\frac{2}{\pi} \frac{\sqrt{2} n_{0}}{(8 \pi)^{d / 2} \sigma}\right)^{1 / 2} t^{-(d+3) / 8}\left[1+\frac{\epsilon}{8}(3 \ln 2-1)+\mathrm{O}\left(\epsilon^{2}\right)\right] .
$$

Using the inequalities

$$
\langle\phi-|\psi|\rangle^{2} \leq\left\langle(\phi-|\psi|)^{2}\right\rangle \leq\left\langle\phi^{2}\right\rangle-\left\langle\psi^{2}\right\rangle=\frac{1}{\lambda}(-\dot{\Phi}(t))
$$

in conjunction with the upper bound $\Phi_{\mathrm{u}}(t)$ as in [4] one can show that the lower bound (70) gives the exact long-time behaviour of the density, i.e. $\Phi(t) \simeq \Phi_{1}(t)$ for $t \rightarrow \infty$.

In three dimensions the random velocity field gives a logarithmic contribution to the density $\Phi(t)$. Using equation (39) one obtains

$$
\Phi(t) \simeq \frac{n_{0}^{1 / 2}}{2 \pi} t^{-3 / 4}\left(2 f \ln \left(\Lambda^{2} t\right)\right)^{-1 / 4} .
$$

\section{Summary and Discussion}

In this paper the effects of a quenched random shear flow on the long-time behaviour of the $\mathrm{A}+\mathrm{B}$-annihilation reaction have been studied. It is well-known that in dimensions

Figure 5. Non-zero contributions to $c_{4}(t)$ at second order in $f(\mathrm{~b}-\mathrm{d})$. The first order diagram (a) vanishes.

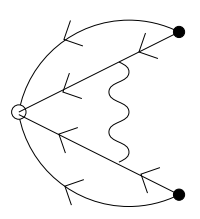

(a)

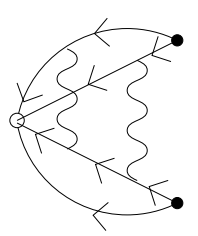

(b)

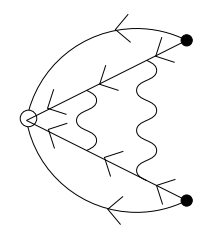

(c)

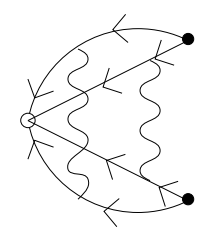

(d) 
$d<3$ a random velocity field gives rise to enhanced diffusion and should, therefore, accelerate the reaction process. Similar to the case of purely diffusive particle motion the system segregates after a short time into regions of purely A or B particles. After this initial stage the density decay is governed by an effective Gaussian distribution for the initial density fluctuations. While a simple model based on anisotropic Lévy walks already yields the correct exponent for the density decay one has to take many-particle correlations generated by the quenched randomness into account in order to calculate the amplitude. We have shown how this is possible in the framework of a systematic expansion in $\epsilon=3-d$ and computed the first order term in this expansion.

In the present paper it was assumed that the diffusion constants $D_{A}, D_{B}$ of $\mathrm{A}$ and $B$ particles are equal and that the hopping rate of both species depends in the same way on $v(\mathbf{y})$. The more general case $D_{A} \neq D_{B}$ with three different disorder couplings $f_{A}$, $f_{B}, f_{A B}$ (instead of $f$ ) can be treated in a similar way at least as long as both species are mobile. For unequal diffusion constants the fields $\phi$ and $\psi$ are coupled already in the Gaussian part of the action. Using similar arguments as in reference [4] it can be shown that this effect changes only the amplitude of $\Phi(t)$.

It would be interesting to check the results of this work by simulations. In order to observe the asymptotic long-time behaviour of a disordered system one has to perform configurational averages unless the system size is very large. In the case of a random shear flow the sample-to-sample fluctuations of the density depend on the linear size $L_{y}$ in the direction perpendicular to the velocity field. Since a diffusing particle covers a distance $l_{D}(t) \sim(D t)^{1 / 2}$ during the time $t$ the density measured at time t represents approximatively $N \sim L_{y} / l_{D}(t)$ independent configurations of the flow. Therefore, lack of self-averaging in a finite sample leads to a relative error of the order $N^{-1 / 2} \sim(D t)^{1 / 4} L_{y}^{-1 / 2}$. In a pure system (without shear flow) the asymptotic power law can be observed at times of the order $D t \sim 10^{5}$ [1]. The above estimate shows that a random system with $L_{y}>1.2 \cdot 10^{5}$ (or an equivalent number of smaller systems) is required to reduce the sample-to-sample fluctuations for $D t \sim 10^{5}$ to less than $5 \%$.

\section{Acknowledgment}

The author thanks J. Cardy, M. Howard and B. P. Lee for useful discussions. This work has been supported by grant Oe199/1-1 of the Deutsche Forschungsgemeinschaft.

\section{References}

[1] Toussaint D and Wilczek F 1983 J. Chem. Phys. 78 2642-7

[2] Kang K and Redner S 1985 Phys. Rev. A 32 435-47

[3] Bramson M and Lebowitz J L 1991 J. Stat. Phys. 62 297-372

[4] Lee B P and Cardy J 1995 J. Stat. Phys. 80 971-1007 
[5] Sokolov I M, Schnörer H and Blumen A 1991 Phys. Rev. A 44 2388-93

[6] Deering W D and West B J 1991 J. Stat. Phys. 65 1247-60

[7] Araujo M, Havlin S, Larralde H and Stanley H E 1992 Phys. Rev. Lett. 68 1791-4

[8] Janowsky S A 1995 Phys. Rev. E 51 1858-60

-1995 Phys. Rev. E 52 2535-9

[9] Ispolatov I, Krapivsky P L and Redner S 1995 Phys. Rev. E 52 2540-5

[10] Zumofen G and Klafter J 1994 Phys. Rev. E 50 5119-22

[11] Howard M J and Barkema G T 1996 Phys. Rev. E 53 5949-56

[12] Sokolov I M and Blumen A 1991 Phys. Rev. Lett. 66 1942-5

[13] 1993 Phys. Lett. A 178 364-8

[14] Matheron G and de Marsily G 1980 Water Resources Res. 16 901-17

[15] Honkonen J 1991 J. Phys. A: Math. Gen. 24 L1235-42

[16] Bouchaud J-P, Georges A, Koplik J, Provata A and Redner S 1990 Phys. Rev. Lett. 64 2503-6

[17] Peliti L 1985 J. Physique 46 1469-83

[18] Lee B P 1994 J. Phys. A: Math. Gen. 27 2633-52

[19] Doi M 1976 J. Phys. A: Math. Gen. 9 1465-77

-1976 J. Phys. A: Math. Gen. 9 1479-95

[20] Grassberger P and Scheunert M 1980 Fortschr. Phys. 28 547-78

[21] ben-Avraham D, Leyvraz F and Redner S 1992 Phys. Rev. A 45 2315-9 